\title{
DNA aptamer raised against advanced glycation end products inhibits melanoma growth in nude mice
}

\author{
Ayako Ojima', Takanori Matsui ${ }^{1}$, Sayaka Maeda' ${ }^{1}$, Masayoshi Takeuchi ${ }^{2}$, Hiroyoshi Inoue ${ }^{3}$, Yuichiro Higashimoto ${ }^{4}$ \\ and Sho-ichi Yamagishi ${ }^{1}$
}

Epidemiological studies have suggested that diabetes is associated with an increased risk of cancer. However, the underlying molecular mechanism remains unclear. We investigated here whether DNA aptamer directed against advanced glycation end products (AGE-aptamer) inhibited melanoma growth in nude mice. G361 melanoma cells were injected intradermally into the upper flank of athymic nude mice. Mice received continuous intraperitoneal infusion $(0.136 \mu \mathrm{g} /$ day) of either AGE-aptamer $(n=9)$ or Control-aptamer $(n=8)$ by an osmotic mini pump. Tumor volume was measured at 4-day interval, and G361 melanoma was excised at day 43 after the aptamer treatment. We further examined the effects of AGE-aptamer on proliferation of AGE-exposed endothelial cells and G361 cells. AGE-aptamer significantly inhibited the in vivo-tumor growth of G361 melanoma. Immunohistochemical and western blotting analyses of G361 melanoma revealed that AGE-aptamer decreased expression levels of proliferating nuclear antigen, CD31 and Mac-3, markers of endothelial cells and macrophages, respectively. AGE-aptamer significantly decreased the number of tumorassociated vessels. AGE, receptor for AGE (RAGE) and vascular endothelial growth factor levels were also reduced in AGE-aptamer-treated G361 melanoma. AGE-aptamer inhibited the AGE-induced proliferation and tube formation of endothelial cells as well as the growth of G361 cells in vitro. The present findings suggest that AGE-aptamer could inhibit the AGE-RAGE axis in G361 melanoma and resultantly suppress the tumor growth in nude mice by blocking the angiogenesis. AGE-aptamer might be a novel therapeutic strategy for preventing the progression of malignant melanoma in diabetes.

Laboratory Investigation (2014) 94, 422-429; doi:10.1038/labinvest.2014.5; published online 10 February 2014

KEYWORDS: AGE; aptamer; diabetes; melanoma; RAGE

Epidemiological studies have suggested that diabetes is associated with an increased risk of various types of cancer. ${ }^{1-5}$ In the Västerbotten Intervention Project of northern Sweden, total cancer risk in women increased with rising plasma levels of fasting and postload glucose, up to a relative risk for the top versus bottom quartile of 1.26 and 1.31, respectively. ${ }^{1}$ Risk of cancer of the pancreas, endometrium, urinary tract and of malignant melanoma was statistically significantly associated with high fasting glucose, which was totally independent on age, smoking and body mass index. ${ }^{1}$ Although the underling molecular mechanisms remain unclear, a nonenzymatic reaction between reducing sugars and the amino groups of proteins, lipids and nucleic acids may have a role in the increased risk for a variety of cancers in diabetes. The process of glycation has been progressed at an accelerated rate under diabetes, leading to the formation and accumulation of advanced glycation end products (AGE). ${ }^{6,7}$ There is accumulating evidence that interaction of AGE with the receptor for AGE (RAGE) contributes to the development and progression of various age- or diabetes-related disorders, including cardiovascular disease, Alzheimer's disease, osteoporosis, cancer growth and metastasis. ${ }^{8-11}$ Indeed, we, along with others, have previously shown that AGE stimulate growth or migration of pancreatic cancer, lung cancer, malignant melanoma and renal cell carcinoma cells in vitro through the interaction with RAGE. ${ }^{12-15}$ Therefore, the AGE-RAGE axis

\footnotetext{
'Department of Pathophysiology and Therapeutics of Diabetic Vascular Complications, Kurume University School of Medicine, Kurume, Japan; ${ }^{2}$ Department of Advanced Medicine, Medical Research Institute, Kanazawa Medical University, Kanazawa, Japan; ${ }^{3}$ Department of Chemistry, Keio University School of Medicine, Kanagawa, Japan and ${ }^{4}$ Department of Medical Biochemistry, Kurume University School of Medicine, Kurume, Japan

Correspondence: Dr S-i Yamagishi, MD, PhD, Department of Pathophysiology and Therapeutics of Diabetic Vascular Complications, Kurume University School of Medicine, 67 Asahi-machi, Kurume 830-0011, Japan.

E-mail: shoichi@med.kurume-u.ac.jp

Received 2 July 2013; revised 25 November 2013; accepted 19 December 2013
} 
may be a therapeutic target for slowing the progression of cancers in patients with diabetes.

Among various cancers, malignant melanoma is one of the most highly invasive and metastatic tumors with an incidence and mortality that have been rapidly increasing in recent years. ${ }^{16,17}$ Although therapeutic advances in the treatment of malignant melanoma have been made with anti-cancer drugs and immunotherapy, these therapeutic options may be limited by considerable side effects. ${ }^{16,17}$ Therefore, novel therapeutic strategies that prevent the growth and progression of melanoma are highly desired in patients with diabetes.

Aptamers are short, single-stranded DNA or RNA molecules that can bind with high affinity and specificity to a wide range of target proteins. ${ }^{18}$ Numerous aptamers have been developed and used in the clinical fields as a tool for modulating the function of various proteins. ${ }^{19,20}$ Pegaptanib, an RNA aptamer directed against vascular endothelial growth factor $_{165}\left(\mathrm{VEGF}_{165}\right)$ isoform, has been shown to be effective in treating choroidal neovascularization in patients with agerelated macular degeneration. ${ }^{19}$ We have recently found that high-affinity DNA aptamer directed against AGE (AGEaptamer) blocks the progression of diabetic nephropathy in an animal model of obese and type 2 diabetes. ${ }^{21}$ Therefore, in this study, we examined whether and how AGE-aptamer could inhibit growth of G361 cells, human malignant melanoma cells, in nude mice. We further investigated the effects of AGE-aptamer on AGE-induced proliferation of G361 and human endothelial cells in vitro.

\section{METHODS AND METHODS Materials}

Bovine serum albumin (BSA) (essentially fatty acid free and essentially globulin free, lyophilized powder) was purchased from Sigma (St Louis, MO, USA). D-glyceraldehyde was purchased from Nakalai Tesque (Kyoto, Japan).

\section{Screening and Modification of AGE-Aptamer (Systematic Evolution of Ligands by EXponential enrichment (SELEX))}

Preparation and selection of DNA aptamers were performed as described previously. ${ }^{21}$ Sequences of AGE-aptamer and control DNA aptamer (Control-aptamer) are as below. AGEaptamer; 5'-CCGAAACCAGACCACCCCACCAAGGCCACT CGGTCGAACCGCCAACACTCACCCCA- ${ }^{\prime}$, and Controlaptamer; 5'-GTTATCTGTCATAGGAACAGTCAGACTCAG CGTCGCAGTTCAGGGCACTTTAGCAC-3'. DNA aptamers are susceptible to degradation by nucleases. ${ }^{21}$ This will limit their applications for real samples, such as blood and tissues. To solve this issue, we modified aptamers with phosphorothioate as described previously. ${ }^{21}$ We confirmed that AGEaptamer, but not Control-aptamer, bound to AGE-modified human serum albumin with a dissociation constant of $1.38 \times 10^{-6} \mathrm{M}$.

\section{Growth of G361 Melanoma in Nude Mice}

Two million G361 cells were injected intradermally into the upper flank of 6-week-old female athymic nude mice (Japan Clea, Tokyo, Japan; $n=17) .{ }^{14}$ Mice received continuous intraperitoneal infusion $(0.136 \mu \mathrm{g} /$ day $)$ of either AGEaptamer $(n=9)$ or Control-aptamer $(n=8)$ by an osmotic mini pump (Alzet osmotic pumps, model 1004, Cupertino, CA, USA) and were maintained under specific pathogen-free conditions as described previously. ${ }^{21}$ Later, the smallest and largest diameters of tumors were measured at 4-day interval with a digital caliper, and tumor volumes were calculated using the following formula: volume $\left(\mathrm{mm}^{3}\right)=(($ smallest diameter $)^{2} \times($ largest diameter $\left.)\right) / 2$ as described previously. ${ }^{14}$ Forty-three days after the continuous intraperitoneal infusion of each aptamer, G361 melanoma was excised for immunohistochemical and western blotting analyses. All experimental procedures were conducted in accordance with the National Institutes Health Guide for Care and Use of Laboratory Animals and were approved by the ethnical committee of Kurume University School of Medicine.

\section{Immunohistochemical Analysis}

Specimens of G361 melanoma were fixed with 4\% paraformaldehyde, embedded in paraffin, sectioned at $4-\mu \mathrm{m}$ intervals and mounted on glass slides. The sections were incubated in $0.3 \%$ hydrogen peroxide methanol for $30 \mathrm{~min}$ to block endogenous peroxidase activity and incubated overnight at $4{ }^{\circ} \mathrm{C}$ with antibodies (Abs) raised against proliferation cell nuclear antigen (PCNA) (Abcam, Cambridge, United Kingdom), CD31 (Santa Cruz Biotechnology, Santa Cruz, CA, USA), Mac-3 (Santa Cruz Biotechnology), AGE, RAGE (Santa Cruz Biotechnology) and VEGF (Santa Cruz Biotechnology) as described previously. ${ }^{22}$ Then the reactions were visualized with a Histofine Simple Stain Mouse MAXPO kit (Nichirei, Tokyo, Japan). Immunoreactivity of each sample was measured by microcomputer-assisted ImageJ.

\section{Western Blotting Analysis}

Twenty micrograms of proteins were extracted from G361 melanoma with lysis buffer, and then separated by SDS-PAGE and transferred to nitrocellulose membranes as described previously. ${ }^{23}$ Membranes were probed with Abs against PCNA, CD31, lysosomal-associated membrane protein 2 (Mac-3) (Abcam), AGE, RAGE, VEGF, $\alpha$-tubulin (Santa Cruz Biotechnology) or $\beta$-actin (Santa Cruz Biotechnology), and then immune complexes were visualized with an enhanced chemiluminescence detection system (Amersham Bioscience, Buckinghamshire, United Kingdom).

\section{Preparation of AGE-BSA}

AGE-BSA was prepared as described previously. ${ }^{24}$ In brief, BSA $(25 \mathrm{mg} / \mathrm{ml})$ was incubated under sterile conditions with $0.1 \mathrm{M}$ glyceraldehyde in $0.2 \mathrm{M} \mathrm{NaPO}_{4}$ buffer ( $\mathrm{pH}$ 7.4) for 7 days. Then unincorporated sugars were removed by PD-10 column chromatography and dialysis against 
phosphate-buffered saline. Control non-glycated BSA was incubated in the same conditions except for the absence of reducing sugars. Preparations were tested for endotoxin using Endospecy ES-20S system (Seikagaku, Tokyo, Japan); no endotoxin was detectable.

\section{Assay of Tumor-Associated Angiogenesis}

After two million G361 cells were injected intradermally into athymic nude mice, mice received continuous intraperitoneal infusion of either AGE-aptamer $(n=9)$ or Control-aptamer $(n=8)$ as described above. On day 43 after the procedure, tumors were excised, and tumor-associated angiogenesis was quantitated by counting the number of vessels in G361 melanoma cells according to the method described by Murata et $a .^{25}$

\section{Preparation of Ab Raised Against Human RAGE (RAGE- Ab)}

RAGE-Ab, which recognizes the amino-acid residues $167-180$ of human RAGE protein, was prepared and used for neutralizing assays as described previously. ${ }^{26}$

\section{G361 Cells}

G361 cells (American Type Culture Collection, Manassas, VA, USA) were maintained in Dulbecco's modified Eagle's medium (DMEM) (Sigma) supplemented with $10 \%$ fetal bovine serum (FBS). G361 cells in DMEM containing 1\% FBS were treated with $1000 \mu \mathrm{g} / \mathrm{ml}$ AGE-BSA or non-glycated BSA for $24 \mathrm{~h}$ in the presence or absence of $2 \mu \mathrm{M}$ AGE-aptamer or Control-aptamer.

\section{Human Umbilical Vein Endothelial Cells (HUVEC)}

HUVEC were cultured in endothelial basal medium supplemented with $2 \% \mathrm{FBS}, 0.4 \%$ bovine brain extracts, $10 \mathrm{ng} / \mathrm{ml}$ human epidermal growth factor and $1 \mu \mathrm{g} / \mathrm{ml}$ hydrocortisone according to the supplier's instructions (Clonetics, San Diego, CA, USA). HUVEC were treated with $100 \mu \mathrm{g} / \mathrm{ml}$ AGE-BSA or non-glycated BSA for $24 \mathrm{~h}$ in the presence or absence of $2 \mu \mathrm{M}$ AGE-aptamer, $2 \mu \mathrm{M}$ Control-aptamer or $5 \mu \mathrm{g} / \mathrm{ml}$ RAGE-Ab. Cell proliferation experiments were carried out in a medium lacking epidermal growth factor and hydrocortisone (assay medium).

\section{Cell Proliferation Assay}

Cell proliferation levels were measured with an electron coupling reagent WST-1-based colorimetric assay according to the supplier's recommendations (Roche Applied Science, Indianapolis, IN, USA).

\section{Tube Formation In Vitro}

Assay medium was incubated with $100 \mu \mathrm{g} / \mathrm{ml}$ non-glycated BSA or AGE-BSA in the presence or absence of $2 \mu \mathrm{M}$ AGE-aptamer or Control-aptamer at $37^{\circ} \mathrm{C}$ for $2 \mathrm{~h}$. Then HUVEC $(1 \times 104$ cells $)$ were seeded on BD Biocoat Cellware Matrigel Basement Membrane Matrix 48-well Plates (BD
Bioscience, Franklin Lakes, NJ, USA) and incubated in the pretreated assay medium. After $3 \mathrm{~h}$, three microscopic fields selected at random from each well $(N=3)$ were photographed, and the lengths of tube-like structures were measured with a microcomputer-assisted ImageJ as described previously. ${ }^{27}$

\section{Statistical Analysis}

All values were presented as means \pm s.e. One-way ANOVA followed by the Scheffe's $F$ test or Student's $t$ test was performed for statistical comparisons; $P<0.05$ was considered significant.

\section{RESULTS}

Effects of AGE-Aptamer on Tumor Growth in Nude Mice We first investigated whether continuous infusion of AGEaptamer could inhibit melanoma growth in nude mice. G361 melanoma cells formed rapidly growing tumors in nude mice, reaching $400-500 \mathrm{~mm}^{3}$ after 43 days. In contrast, continuous intraperitoneal injection of AGE-aptamer significantly inhibited the in vivo tumor growth of G361 melanoma over an observation period of up to 43 days (Figure 1).

\section{Effects of AGE-Aptamer on PCNA, CD31, Tumor- Associated Angiogenesis and Mac-3 Levels in G361 Melanoma}

Immunohistochemical detection of proliferating cells revealed that ratio of PCNA-positive cell nuclei to total cell nuclei was lower in AGE-aptamer-treated G361 melanoma (Figure 2a). Furthermore, compared with Control-aptamer-treated mice, PCNA levels in G361 melanoma of AGE-aptamer-treated mice were significantly decreased (Figure 2a). However, apoptotic cell death of G361 melanoma was not increased by the treatment of AGE-aptamer (data not shown).

Tumor-associated angiogenesis and macrophage infiltration have been known to contribute to tumor growth and expansion in vivo. ${ }^{28,29}$ Furthermore, VEGF not only has a crucial role in tumor angiogenesis and expansion but it could also evoke inflammatory reactions in tumors. ${ }^{30-35}$ As we have previously shown that VEGF is induced by engagement of RAGE with AGE, ${ }^{24,26}$ we studied the effects of AGE-aptamer on angiogenesis and macrophage infiltration in G361 melanoma and then investigated the involvement of AGE-RAGE-VEGF axis in anti-tumor actions of aptamer. For this, we first examined the effects of AGE-aptamer on expression levels of CD31 and Mac-3, markers of capillary endothelial cells and macrophages, respectively. ${ }^{30}$ Immunohistochemical and western blotting analyses revealed that AGE-aptamer treatment significantly suppressed angiogenesis and macrophage infiltration in the tumors of nude mice (Figures $2 \mathrm{~b}$ and d). Furthermore, AGE-aptamer significantly decreased the number of tumor-associated vessels (Figure 2c). 

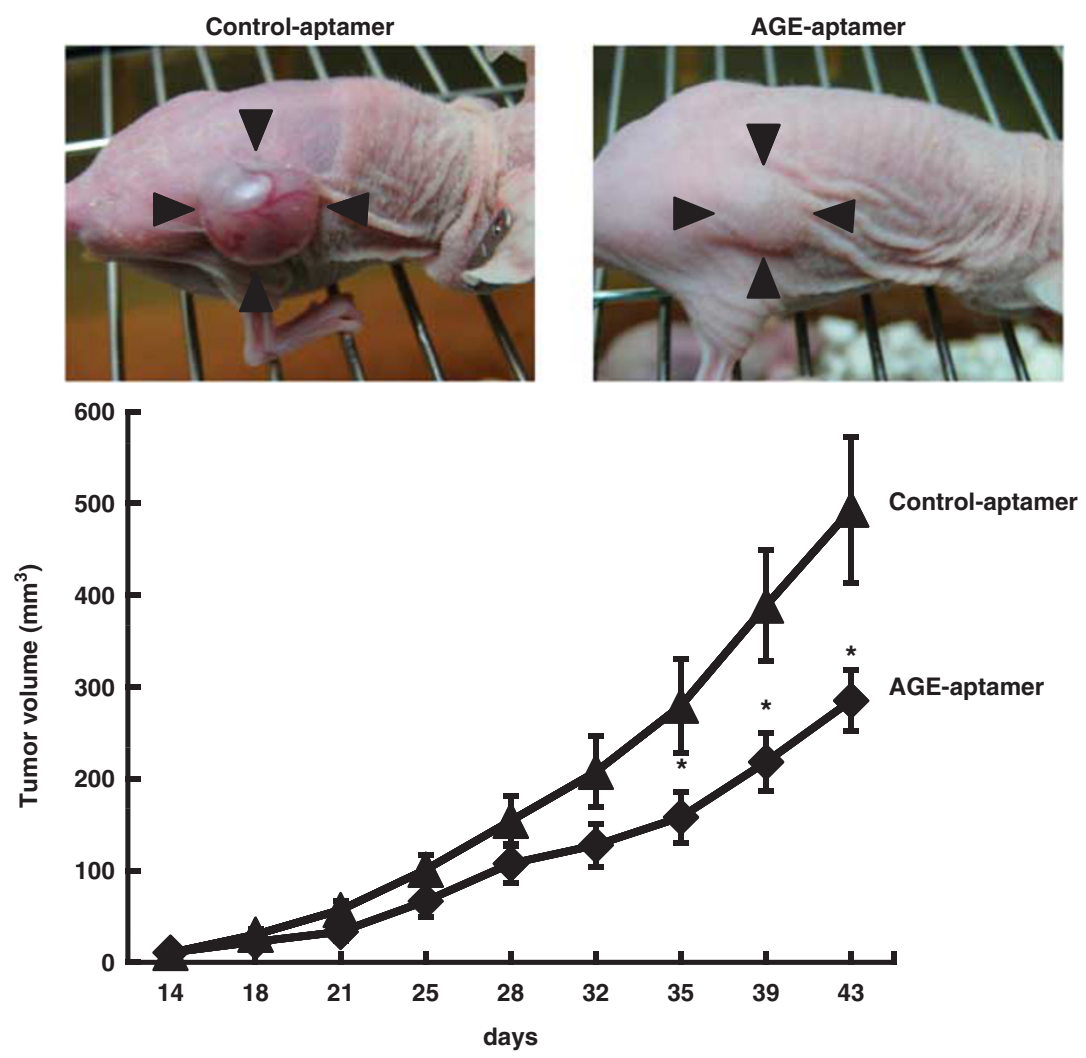

Figure 1 Effects of AGE-aptamer on tumor growth in nude mice. Two million G361 cells were injected intradermally into the upper flank of 6-week-old female athymic nude mice $(n=17)$. Mice received continuous intraperitoneal infusion $(0.136 \mu \mathrm{g} /$ day) of either AGE-aptamer $(n=9)$ or Control-aptamer $(n=8)$ by an osmotic mini pump. Then tumor volume was measured at 4-day interval. Upper panel shows typical photographs of G361 xenograft at 43 days after each aptamer injection. Arrowheads indicate tumors. ${ }^{*} P<0.05$ compared with the value of Control-aptamer-treated mice.

\section{Effects of AGE-Aptamer on Expression Levels of AGE, RAGE and VEGF in G361 Melanoma}

We next examined the AGE, RAGE and VEGF expression levels in the tumors. Both AGE-modified proteins and their receptor RAGE expression in G361 melanoma were significantly decreased by the treatment of AGE-aptamer. Moreover, compared with Control-aptamer, continuous administration of AGE-aptamer significantly suppressed VEGF expression levels in G361 melanoma as well (Figures 3a-c).

\section{Effects of AGE-Aptamer on Proliferation and Tube Formation of HUVEC}

As shown in Figure 4a, AGE significantly increased proliferation of HUVEC, which was blocked by the treatment of AGE-aptamer or RAGE-Ab. Furthermore, AGE-aptamer, but not Control-aptamer, significantly blocked the AGE-induced increase in tube formation of HUVEC in vitro (Figure $4 \mathrm{~b}$ ).

\section{Effects of AGE-Aptamer on Proliferation of G361 Cells} As shown in Figure 4c, AGE significantly increased proliferation of G361 melanoma cells in vitro, which was completely prevented by the treatment with AGE-aptamer.

\section{DISCUSSION}

In this study, we have found for the first time that (1) longterm continuous intraperitoneal injection of AGE-aptamer inhibits the in vivo-tumor growth of G361 melanoma in nude mice, (2) number of proliferating cells in G361 melanoma is significantly decreased by the treatment of AGE-aptamer, whereas AGE-aptamer does not affect apoptotic cell death of tumor cells, (3) AGE-aptamer significantly suppresses tumorassociated angiogenesis in nude mice, (4) AGE-aptamer significantly decrease expression levels of AGE, RAGE and VEGF in G361 melanoma, (5) AGE stimulate the growth and tube formation of HUVEC, both of which are significantly inhibited by the treatment of AGE-aptamer, but not Controlaptamer, and (6) AGE-aptamer completely prevents the AGE-induced G361 melanoma cell proliferation in vitro. These observations demonstrate that AGE-aptamer might inhibit the AGE-RAGE axis in G361 melanoma and resultantly suppress the tumor growth in nude mice by blocking the angiogenesis. Given the accelerated formation and accumulation of AGE in diabetes, ${ }^{6,7}$ our present findings suggest that AGE-aptamer might be a novel therapeutic strategy for preventing the progression of malignant melanoma in diabetes. 

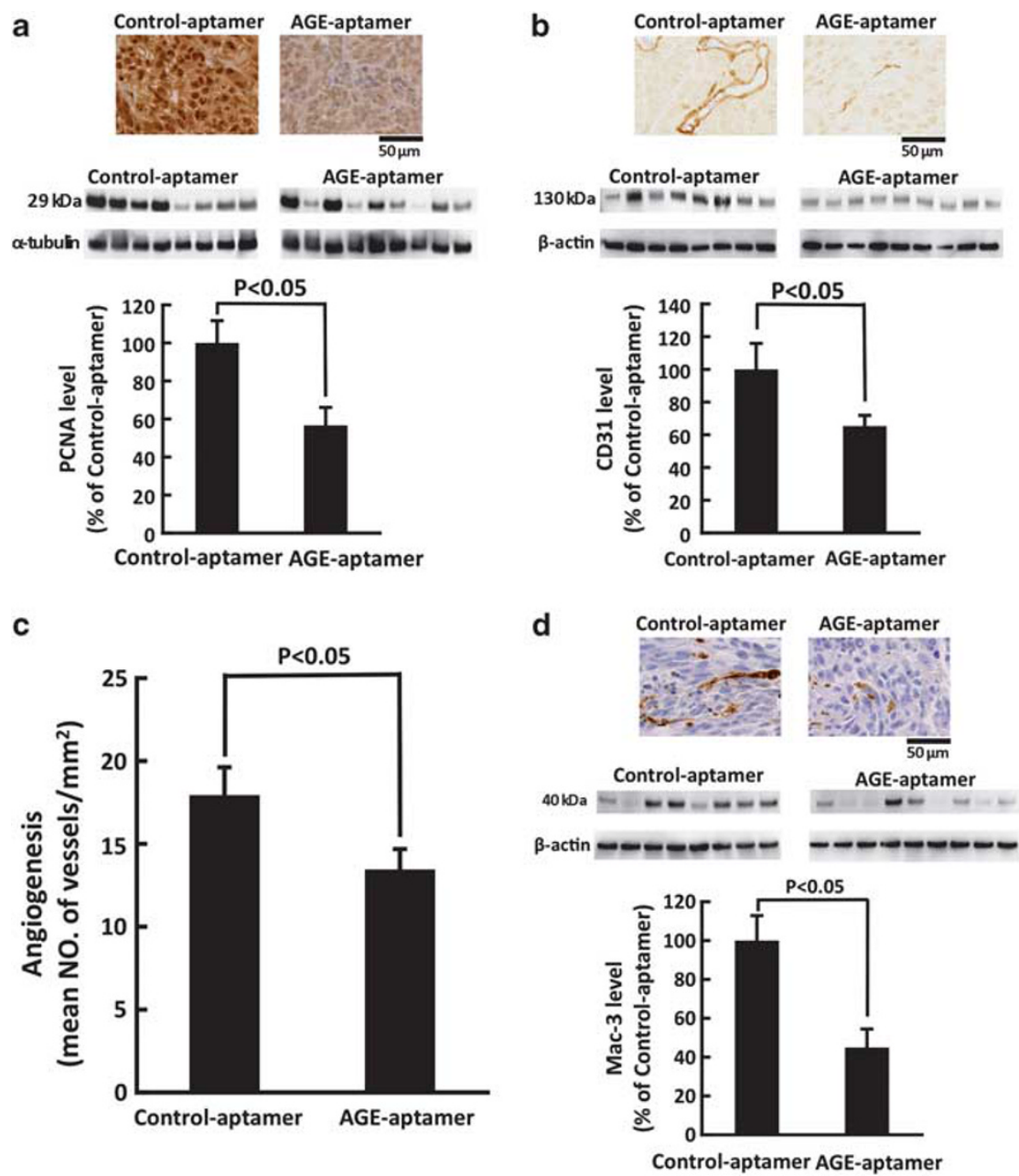

Figure 2 Effects of AGE-aptamer on expression levels of PCNA (a), CD31 (b), tumor-associated angiogenesis (c) and Mac-3 (d) in G361 melanoma. (a, b and $\mathbf{d}$ ) Upper panel shows representative photographs of each immunostaining. Middle panel shows the bands of western blotting. Quantitative data are shown in lower panel. Data were normalized by the intensity of $\alpha$-tubulin- (a) or $\beta$-actin-derived signals $(\mathbf{b}$, $\mathbf{d}$ ) and related to the value of Control-aptamer-treated mice. (c) Number of vessels in tumors was counted. Panel $\mathbf{b}$ had no counterstaining. $\beta$-actin bands in Figure $2 \mathrm{~b}$ and Figure $3 \mathrm{~b}$ are same because membrane was stripped and reprobed.

Several lines of evidence implicate VEGF as the key angiogenic factor involved in melanoma growth and metastasis. ${ }^{30-33}$ Furthermore, VEGF acts as a pro-inflammatory cytokine; it could induce macrophage infiltration into tumors as well. ${ }^{34,35}$ Indeed, VEGF expression levels are associated with angiogenesis and macrophage infiltration, the extent of which being correlated with melanoma prognosis. ${ }^{30-33}$ Therefore, our present study suggests that AGEaptamer might inhibit tumor-associated angiogenesis and macrophage infiltration by blocking the VEGF expression in G361 melanoma.

We have previously shown that the AGE-RAGE interaction stimulates growth and tube formation of endothelial cells through the autocrine production of VEGF. ${ }^{36}$ Moreover, AGE also induce VEGF expression in tumor cells via the interaction with RAGE. ${ }^{26,37}$ In this study, expression levels of AGE, RAGE and VEGF were suppressed mainly in tumor cells. So, blockade of the AGE-RAGE axis by AGE-aptamer in G361 tumor cells might contribute to the suppression of VEGF expression. However, involvement of other cell types cannot be totally ruled out.

We have very recently found that AGE-aptamer used here not only inhibits the binding of AGE to RAGE and subsequently suppresses its downstream signaling in vitro but could also accelerate the degradation rate of AGE and reduce their accumulation in diabetic mice. ${ }^{21}$ As AGE-RAGE interaction induces RAGE expression in a variety of cells, which could promote the formation of AGE again, ${ }^{8,9}$ AGEaptamer may reduce both AGE and RAGE expression in G361 melanoma by blocking the positive feedback loop between AGE and RAGE axis.

In this study, as the case in HUVEC, AGE-aptamer inhibited the proliferation of AGE-exposed G361 cells in vitro. Furthermore, we have previously shown that 
a
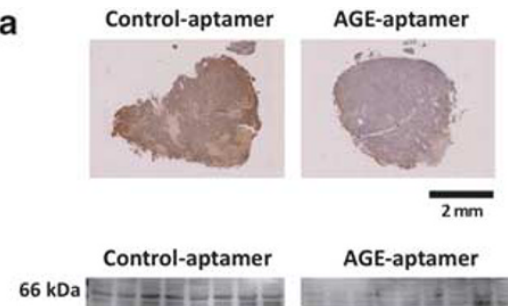

AGE-aptamer
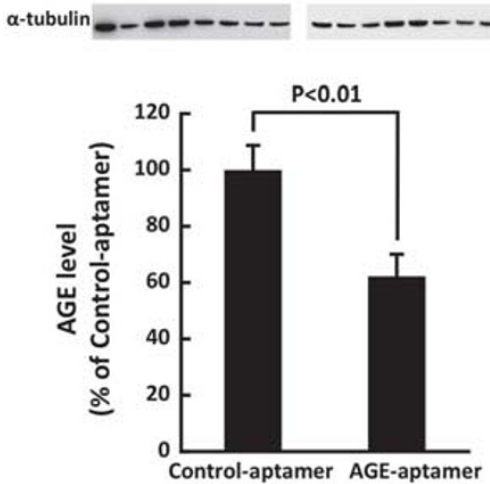

b
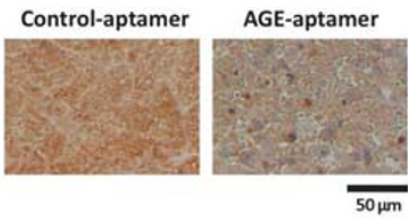

Control-aptamer

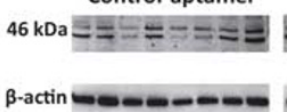

AGE-aptame
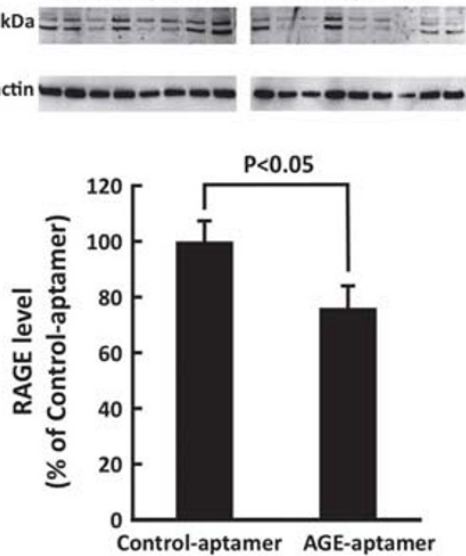

C
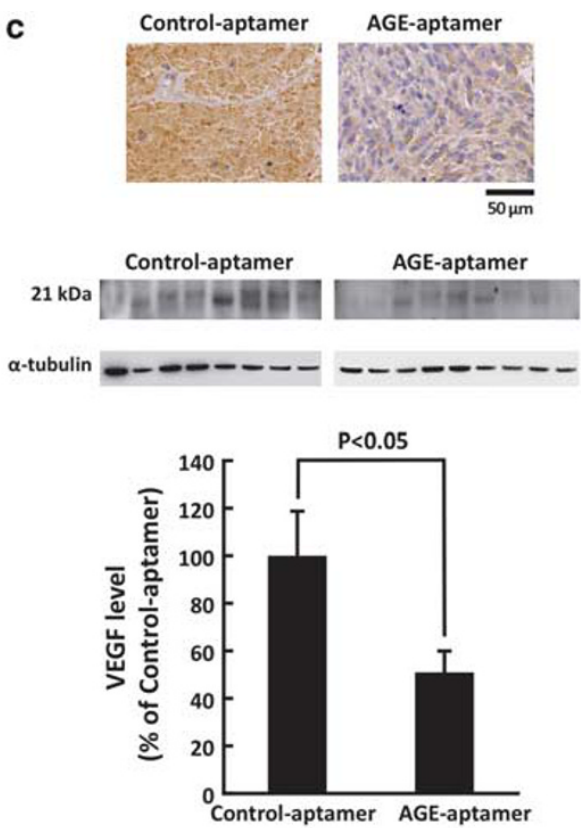

Figure 3 Effects of AGE-aptamer on expression levels of AGE, RAGE and VEGF in G361 melanoma. (a-c) Upper panel shows representative photographs of each immunostaining. Middle panel shows the bands of western blotting. Quantitative data are shown in lower panel. Data were normalized by the intensity of $\alpha$-tubulin- $(\mathbf{a}, \mathbf{c})$ or $\beta$-actin-derived signals (b) and related to the value of Control-aptamer-treated mice. $\alpha$-tubulin bands in panels $\mathbf{a}$ and $\mathbf{c}$ are same because membrane was stripped and reprobed.

a

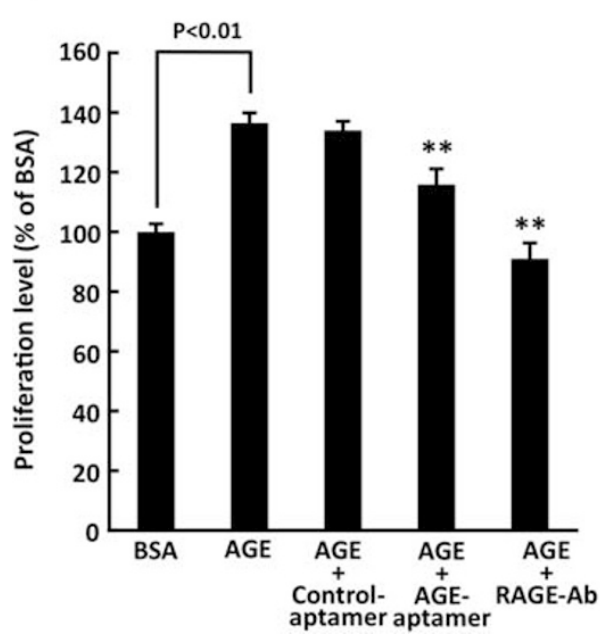

b
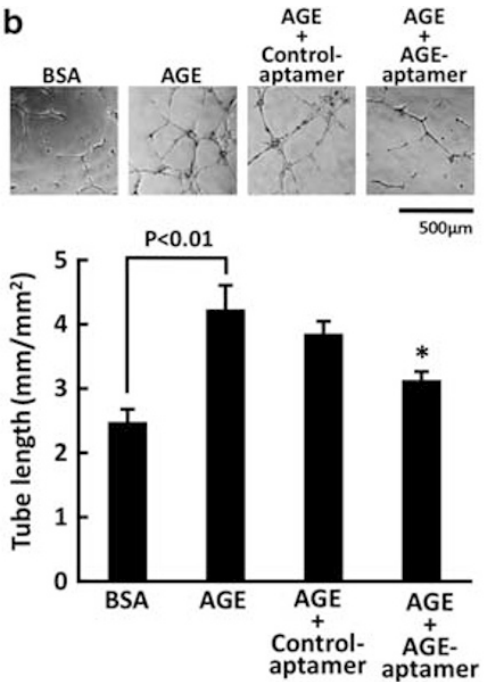

C

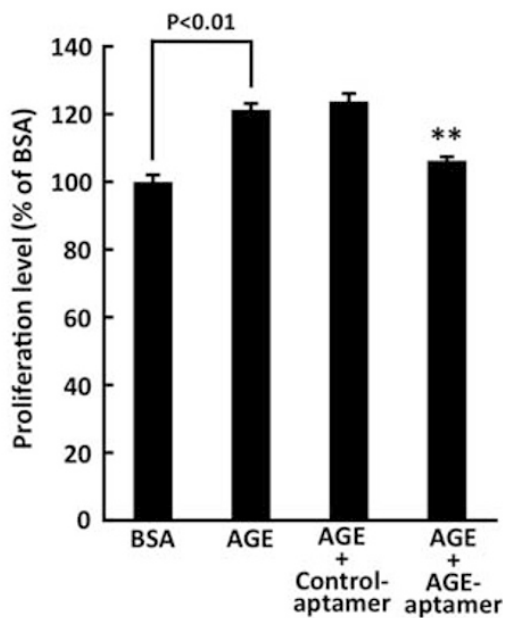

Figure 4 Effects of AGE-aptamer on HUVEC and G361 cell proliferation. (a) HUVEC were treated with $100 \mu \mathrm{g} / \mathrm{ml} \mathrm{AGE-BSA} \mathrm{or} \mathrm{non-glycated} \mathrm{BSA} \mathrm{for} 24 \mathrm{~h}$ in the presence or absence of $2 \mu \mathrm{M}$ AGE-aptamer, $2 \mu \mathrm{M}$ Control-aptamer or $5 \mu \mathrm{g} / \mathrm{ml}$ RAGE-Ab. (b) Assay medium was incubated with $100 \mu \mathrm{g} / \mathrm{ml}$ nonglycated BSA or AGE-BSA in the presence or absence of $2 \mu \mathrm{M}$ AGE-aptamer or Control-aptamer at $37^{\circ} \mathrm{C}$ for $2 \mathrm{~h}$. Then HUVEC were seeded on Matrigelcoated wells and incubated in the pretreated assay medium. After $3 \mathrm{~h}$, nine microscopic fields selected at random were photographed, and the lengths of tube-like structures were measured with a microcomputer-assisted ImageJ. (c) G361 cells were treated with $1000 \mu \mathrm{g} / \mathrm{ml}$ AGE-BSA or non-glycated BSA for $24 \mathrm{~h}$ in the presence or absence of $2 \mu \mathrm{M}$ AGE-aptamer or Control-aptamer. Cell proliferation levels were measured with an electron coupling reagent WST-1-based colorimetric assay. Single $\left(^{*}\right)$ and double asterisks $\left(^{* *}\right)$ indicate $P<0.05$ and $P<0.01$ compared with the value of AGE alone, respectively. (a) $N=18-36$ per group. (b) Three microscopic fields selected at random from each well ( $N=3$ per group) were photographed. (c) $N=5$ per group.

RAGE-Ab completely inhibits the AGE-induced proliferation of G361 cells in vitro. ${ }^{14}$ Therefore, AGE-aptamer might partly work as an anti-melanoma agent by directly acting on G361 melanoma cells. In support of this speculation, we have previously shown that AGE-RAGE interaction stimulates the growth of cultured breast cancer cells. ${ }^{26}$ 
Recent epidemiological study has shown that the increased risk of cancer deaths is lost after adjustment for glycated hemoglobin levels, while the positive association between cancer mortality and diabetes remained significant after adjusting for other risk factors. ${ }^{5}$ These observations suggest that long-term hyperglycemic exposure may explain the link between increased cancer deaths and diabetes. Our present findings suggest that the blockade of AGE-RAGE axis by AGE-aptamer might be a novel therapeutic target of melanoma in patients with diabetes.

\section{LIMITATIONS}

Under diabetic conditions, various metabolic and hemodynamic pathways are activated. ${ }^{8}$ To directly examine the role of AGE-RAGE axis and therapeutic implication of AGE-aptamer in melanoma growth, we used non-diabetic nude mice as a host for the present experiments, although glucose levels were usually normal and AGE-RAGE axis could not be enhanced in these animals. It would be interesting to study further whether melanoma growth is accelerated if these mice will become diabetes.

In this study, continuous intraperitoneal injection of AGEaptamer did not induce apoptotic cell death of G361 melanoma (data not shown). Combination therapy with other anti-cancer agents that sensitize melanoma cells to apoptosis might circumvent this limitation when it comes to applying AGE-aptamer as a therapeutic modality.

\section{ACKNOWLEDGMENTS}

This work was supported in part by MEXT-Supported Program for the Strategic Research Foundation at Private Universities, the Ministry of Education, Culture, Sports, Science and Technology (MEXT) (SY) and by Grants-in-Aid for Scientific Research (B) 22390111 (SY) from the Ministry of Education, Culture, Sports, Science, and Technology of Japan.

\section{DISCLOSURE/CONFLICT OF INTEREST}

The authors declare no conflict of interest.

1. Stattin $P, B j o r ~ O$, Ferrari $P$, et al. Prospective study of hyperglycemia and cancer risk. Diabetes Care 2007;30:561-567.

2. Vigneri $P$, Frasca F, Sciacca $L$, et al. Diabetes and cancer. Endocr Relat Cancer 2009;16:1103-1123.

3. Rousseau $M$, Parent $M$, Pollak $M$, et al. Diabetes mellitus and cancer risk in a population-based case-control study among men from Montreal, Canada. Int J Cancer 2006;118:2105-2109.

4. Kuriki K, Hirose K, Tajima K. Diabetes and cancer risk for all and specific sites among Japanese men and women. Eur J Cancer Prev 2007;16: 83-89.

5. Emerging Risk Factors CollaborationSeshasai SR, Kaptoge S, Thompson $\mathrm{A}$, et al. Diabetes mellitus, fasting glucose, and risk of cause-specific death. N Engl J Med 2011;364:829-841.

6. Rahbar S. The discovery of glycated hemoglobin: a major event in the study of nonenzymatic chemistry in biological systems. Ann NY Acad Sci 2005;1043:9-19.

7. Stitt AW, Bucala R, Vlassara $H$. Atherogenesis and advanced glycation: promotion, progression, and prevention. Ann NY Acad Sci 1997;811: 115-127.

8. Yamagishi S, Imaizumi T. Diabetic vascular complications: pathophysiology, biochemical basis and potential therapeutic strategy. Curr Pharm Des 2005;11:2279-2299.
9. Ramasamy R, Yan SF, Schmidt AM. The diverse ligand repertoire of the receptor for advanced glycation endproducts and pathways to the complications of diabetes. Vascul Pharmacol 2012;57:160-167.

10. Takeuchi M, Yamagishi S. Involvement of toxic AGEs (TAGE) in the pathogenesis of diabetic vascular complications and Alzheimer's disease. J Alzheimers Dis 2009;16:845-858.

11. Yamagishi $\mathrm{S}$. Role of advanced glycation end products (AGEs) in osteoporosis in diabetes. Curr Drug Targets 2011;12:2096-2102.

12. Yamamoto Y, Yamagishi S, Hsu CC, Yamamoto H. Advanced glycation endproducts-receptor interactions stimulate the growth of human pancreatic cancer cells through the induction of platelet-derived growth factor-B. Biochem Biophys Res Commun 1996;222:700-705.

13. Takino J, Yamagishi S, Takeuchi M. Cancer malignancy is enhanced by glyceraldehyde-derived advanced glycation end-products. J Oncol 2010; 739852; doi:10.1155/2010/739852.

14. Abe R, Shimizu $T$, Sugawara $H$, et al. Regulation of human melanoma growth and metastasis by AGE-AGE receptor interactions. J Invest Dermatol 2004;122:461-467.

15. Miki S, Kasayama S, Miki Y, et al. Expression of receptors for advanced glycosylation end products on renal cell carcinoma cells in vitro. Biochem Biophys Res Commun 1993;196:984-989.

16. Atallah E, Flaherty L. Treatment of metastatic malignant melanoma. Curr Treat Options Oncol 2005;6:185-193.

17. Liu LS, Colegio OR. Molecularly targeted therapies for melanoma. Int J Dermatol 2013;52:523-530.

18. Bock LC, Griffin LC, Latham JA, et al. Selection of single-stranded DNA molecules that bind and inhibit human thrombin. Nature 1992;355:564-566.

19. Gragoudas ES, Adamis AP, Cunningham ET, et al. Group VISiONCT: Pegaptanib for neovascular age-related macular degeneration. N Engl J Med 2004;351:2805-2816.

20. Jilma-Stohlawetz P, Gilbert JC, Gorczyca ME, et al. A dose ranging phase $\mathrm{I} / \mathrm{II}$ trial of the von Willebrand factor inhibiting aptamer ARC1779 in patients with congenital thrombotic thrombocytopenic purpura. Thromb Haemost 2011;106:539-547.

21. Kaida Y, Fukami K, Matsui T, et al. DNA aptamer raised against AGEs blocks the progression of experimental diabetic nephropathy. Diabetes 2013;62:3241-3250.

22. Matsui $T$, Nishino $Y$, Takeuchi $M$, et al. Vildagliptin blocks vascular injury in thoracic aorta of diabetic rats by suppressing advanced glycation end product-receptor axis. Pharmacol Res 2011;63:383-388.

23. Yoshida $\mathrm{Y}$, Yamagishi $\mathrm{S}$, Matsui $\mathrm{T}$, et al. Protective role of pigment epithelium-derived factor (PEDF) in early phase of experimental diabetic retinopathy. Diabetes Metab Res Rev 2009;25:678-686.

24. Yamagishi S, Nakamura K, Matsui T, et al. Pigment epithelium-derived factor inhibits advanced glycation end product-induced retinal vascular hyperpermeability by blocking reactive oxygen speciesmediated vascular endothelial growth factor expression. J Biol Chem 2006;281:20213-20220.

25. Murata J, Saiki I, Makabe T, et al. Inhibition of tumor-associated angiogenesis by sulfated chitin derivatives. Cancer Res 1991;51:22-26.

26. Ishibashi $Y$, Matsui T, Takeuchi $M$, et al. Metformin inhibits advanced glycation end products (AGEs)-induced growth and VEGF expression in MCF-7 breast cancer cells by suppressing AGEs receptor expression via AMP-activated protein kinase. Horm Metab Res 2013;45:387-390.

27. Kujawaski $M$, Kortylewski $M$, Lee $H$, et al. Stat3 mediates myeloid celldependent tumor angiogenesis in mice. J Clin Invest 2008;118:3367-3377.

28. Park SJ, Kim HS, Yang HM, et al. Thalidomide as a potent inhibitor of neointimal hyperplasia after balloon injury in rat carotid artery. Arterioscler Thromb Vasc Biol 2004;24:885-891.

29. Volpert OV, Zaichuk T, Zhou W, et al. Inducer-stimulated Fas targets activated endothelium for destruction by anti-angiogenic thrombospondin-1 and pigment epithelium-derived factor. Nat Med 2002;8:349-357.

30. Yamagishi $S$, Abe R, Inagaki $Y$, et al. Minodronate, a newly developed nitrogen-containing bisphosphonate, suppresses melanoma growth and improves survival in nude mice by blocking vascular endothelial growth factor signaling. Am J Pathol 2004;165:1865-1874.

31. Senger DR, van de Water L, Brown LF, et al. Vascular permeability factor in tumor biology. Cancer Metastasis Rev 1993;12:303-324.

32. Torisu $H$, Ono $M$, Furue $M$, et al. Macrophage infiltration correlates with tumor stage and angiogenesis in human malignant melanoma: possible involvement of TNFalpha and IL-1alpha. Int J Cancer 2000;85:182-188. 
33. Salven $P$, Heikkila $P$, Joensuu $H$. Enhanced expression of vascular endothelial growth factor in metastatic melanoma. $\mathrm{Br} J$ Cancer 1997;76:930-934.

34. Vicioso L, Gonzalez FJ, Alvarez M, et al. Elevated serum levels of vascular endothelial growth factor are associated with tumor-associated macrophages in primary breast cancer. Am J Clin Pathol 2006;125:111-118.

35. Tsutsui S, Yasuda K, Suzuki K, et al. Macrophage infiltration and its prognostic implications in breast cancer: the relationship with VEGF expression and microvessel density. Oncol Rep 2005;14:425-431.
36. Yamagishi Si, Yonekura H, Yamamoto Y, et al. Advanced glycation end products-driven angiogenesis in vitro. Induction of the growth and tube formation of human microvascular endothelial cells through autocrine vascular endothelial growth factor. J Biol Chem 1997;272: 8723-8730.

37. Takino J, Yamagishi S, Takeuchi M. Glycer-AGEs-RAGE signaling enhances the angiogenic potential of hepatocellular carcinoma by upregulating VEGF expression. World J Gastroenterol 2012;18: 1781-1788. 